\title{
Bronchoconstriction in Response to Deep Inhalation During Spirometry Testing
}

\author{
Jeffrey M Haynes RRT RPFT
}

\section{Introduction}

In healthy individuals, airway resistance has an inverse relationship with lung volume. ${ }^{1}$ Moreover, deep inhalation is thought to maintain airway smooth muscle homeostasis and to possess bronchodilating and bronchoprotective properties against challenge agents..$^{2-6}$ The bronchodilatory effect of deep inhalation is clearly impaired in asthma patients $2,7,8$; however, the bronchoprotective properties of deep inhalation against challenge test agonists (eg, methacholine) remain robust in many asthma patients with mild airway hyper-responsiveness. ${ }^{5,6}$ In some asthma patients, however, deep inhalation can produce paradoxical bronchoconstriction. ${ }^{5,9,10}$ This case describes a patient who developed significant bronchoconstriction in response to deep inhalation during spirometry testing.

\section{Case Summary}

A 49-y-old white male presented to the pulmonary function laboratory for spirometry, specific airway conductance $\left(\mathrm{sG}_{\mathrm{aw}}\right)$, lung volume testing, and methacholine challenge. The patient had smoked one package of cigarettes/d for $20 \mathrm{y}$ until he quit $1 \mathrm{y}$ before testing. He reported a history of childhood asthma, which had been quiescent throughout his adult life. However, in recent months, the patient reported increasing wheezing and chest tightness after exercise. He noted that symptomatic episodes seemed to occur more frequently following outdoor exercise in cold air. The local temperature on the day of testing was

Mr Haynes is affiliated with the Pulmonary Function Laboratory, St Joseph Hospital, Nashua, New Hampshire.

Mr Haynes has disclosed no conflicts of interest.

Correspondence: Jeffrey M Haynes RRT RPFT, Pulmonary Function Laboratory, St Joseph Hospital, 172 Kinsley Street, Nashua, NH 03060. E-mail: jhaynes@sjhnh.org.

DOI: $10.4187 /$ respcare.03995 $12^{\circ} \mathrm{F}$. According to the patient, a therapeutic trial of albuterol via metered-dose inhaler ameliorated both symptom frequency and severity. An additional asthma risk factor was significant atopy with sensitivities to cats, dogs, horses, pollen, trees, and grass.

On the day of testing, pulmonary function instrumentation passed calibration verifications, and there had been no recent issues with instrument functionality or biologic control testing. The patient demonstrated excellent spirometry technique without evidence of submaximal lung inflation before forced exhalation; however, a progressive decline in the FVC and $\mathrm{FEV}_{1}$ was observed (Table 1). ${ }^{11}$ The patient developed some coughing and reported mild chest tightness. In addition to the progressive decline in numerical values and accompanying symptoms, the emergence of more concave expiratory flow-volume loops further strengthened the suspicion of paradoxical bronchoconstriction in response to the deep inhalation required in spirometry testing. The flow-volume loops from efforts 1 and 6 are superimposed in Figure 1. All spirometry efforts satisfied American Thoracic Society/European Respiratory Society acceptability criteria; however, repeatability criteria were not satisfied as an apparent consequence of progressive bronchoconstriction. ${ }^{12}$

Typically, the largest $\mathrm{FVC}$ and $\mathrm{FEV}_{1}$ should be reported as the best-effort values ${ }^{12}$; however, in this case, it was decided that the largest of the last 3 efforts (effort 6; see Table 1) should be reported as the best effort. The data from all efforts and the technologist's suspicion of deep inhalation-induced bronchoconstriction were shared with the interpreting physician. The rationale for this decision was as follows: Paradoxical bronchoconstriction from deep inhalation was suspected, and the yet-to-be-performed lung volumes and $\mathrm{sG}_{\mathrm{aw}}$ testing should be linked to data representative of the current state of ventilation. The state of ventilation that accompanied effort 1 no longer existed, and coupling lung volume and $\mathrm{sG}_{\mathrm{aw}}$ data to a then-nonexistent milieu might be misleading and potentially affect test interpretation. The reported baseline spirometry, lung volume, and $\mathrm{sG}_{\mathrm{aw}}$ data via whole-body plethysmography are listed in Table 2. Lung volume data indicated signifi- 
Table 1. Serial FVC and $\mathrm{FEV}_{1}$ Measurements During Baseline Spirometry Testing

\begin{tabular}{|c|c|c|c|c|c|c|c|c|}
\hline Effort & FVC (L) & $\%$ Predicted $*$ & Z Score* & Change (\%) & $\mathrm{FEV}_{1}(\mathrm{~L})$ & $\%$ Predicted $*$ & Z Score* & Change (\%) \\
\hline 1 & 5.05 & 92 & -0.61 & NA & 3.28 & 76 & -1.84 & NA \\
\hline 2 & 4.98 & 91 & -0.71 & -1 & 3.09 & 72 & -2.18 & -6 \\
\hline 3 & 4.60 & 84 & -1.23 & -9 & 2.85 & 66 & -2.61 & -13 \\
\hline 4 & 4.49 & 82 & -1.38 & -11 & 2.63 & 61 & -3.01 & -20 \\
\hline 5 & 4.42 & 80 & -1.47 & -12 & 2.62 & 61 & -3.02 & -20 \\
\hline 6 & 4.60 & 84 & -1.23 & -9 & 2.74 & 64 & -2.81 & -16 \\
\hline 7 & 4.36 & 79 & -1.56 & -14 & 2.54 & 60 & -3.08 & -22 \\
\hline
\end{tabular}

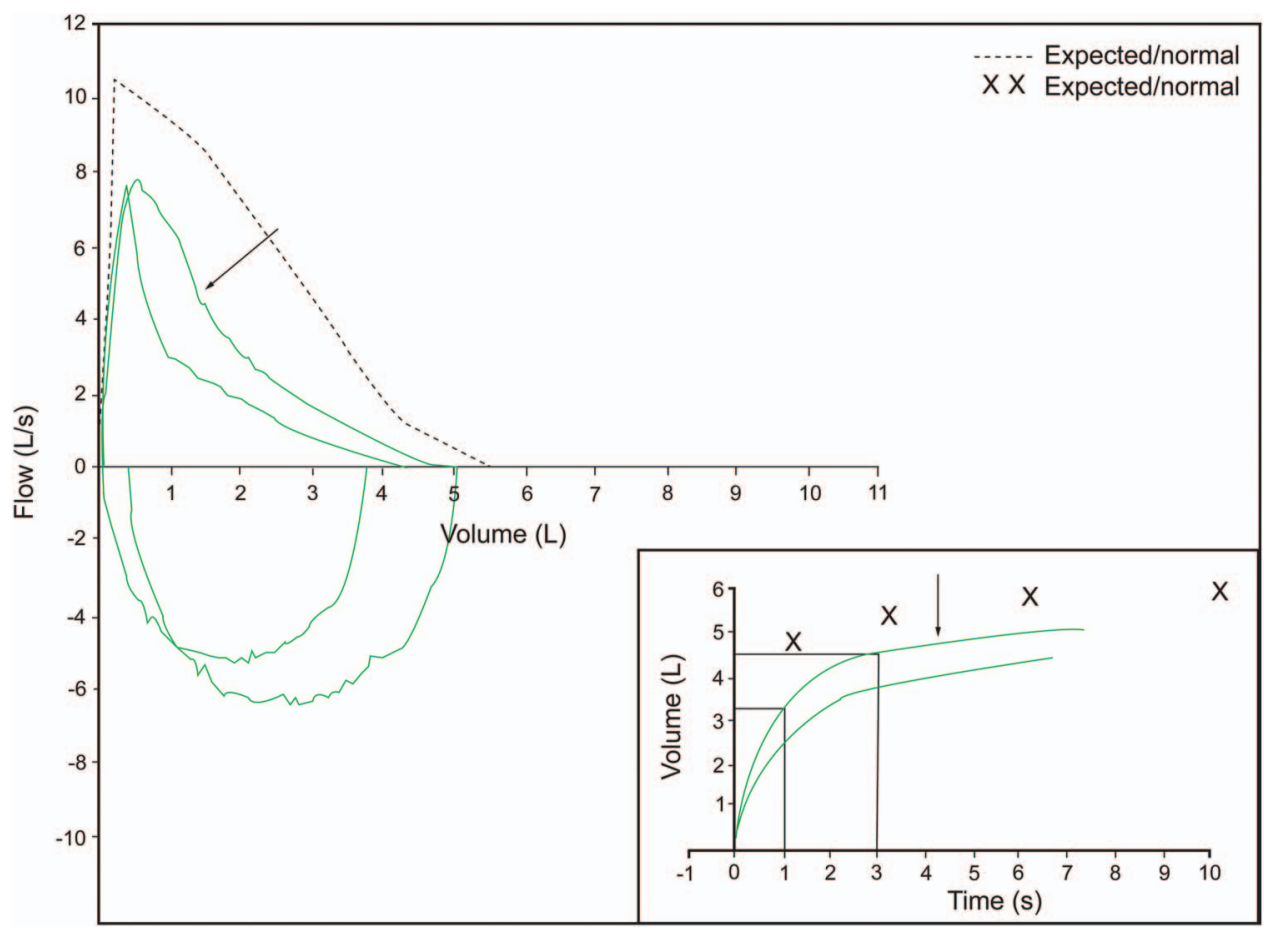

Fig. 1. Superimposed flow-volume loops and volume-time curves from spirometry effort 1 (indicated by arrow) and effort 6 . Effort 6 shows lower flows and volumes compared with effort 1.

cant air-trapping with a residual-volume-to-total-lungcapacity ratio of $41 \%$. In addition, the $\mathrm{sG}_{\mathrm{aw}}$ was below the lower limit of the normal range.

Because of baseline obstruction, the methacholine challenge test was cancelled per laboratory protocol, and bronchodilators $(2.5 \mathrm{mg}$ of albuterol and $0.5 \mathrm{mg}$ of ipratropium via small-volume nebulizers) were administered. The patient demonstrated a significant response to bronchodilators with complete reversal of air-trapping and normalization of the FVC, $\mathrm{FEV}_{1}$, and $\mathrm{sG}_{\mathrm{aw}}$ (see Table 2). The pre-bronchodilator and post-bronchodilator flow-volume loops are superimposed in Figure 2. It is noteworthy that even if the highest $\mathrm{FEV}_{1}$ value (3.28 L, effort 1) had been chosen as the best effort, a $24 \%$ increase in $\mathrm{FEV}_{1}$ follow- ing bronchodilator administration would have been reported.

\section{Discussion}

Spirometric indices are measured to assess lung mechanics and structure via inverse modeling (ie, predict structure from function). ${ }^{13}$ Individuals who perform and interpret spirometry tests need to be aware that the deep inhalation required in the test can affect the existing functional state both positively and negatively. Deep inhalation during spirometry testing has the potential to dilate or constrict the airways and protect against bronchial challenge agents (eg, methacholine). ${ }^{2-10}$ A lack of appreciation 
Table 2. Pulmonary Function Data Pre-Bronchodilator and Post-Bronchodilator

\begin{tabular}{|c|c|c|c|c|c|c|c|c|}
\hline & \multicolumn{4}{|c|}{ Pre-Bronchodilator } & \multicolumn{4}{|c|}{ Post-Bronchodilator } \\
\hline & Actual & $\begin{array}{l}\text { Lower Limit of } \\
\text { the Normal Range }\end{array}$ & $\%$ Predicted & Z Score & Actual & $\%$ Predicted & Z Score & Change (\%) \\
\hline FVC (L) & 4.60 & 4.3 & 84 & -1.2 & 5.85 & 106 & 0.48 & +27 \\
\hline $\mathrm{FEV}_{1}(\mathrm{~L})$ & 2.74 & 3.4 & 64 & -2.8 & 4.06 & 94 & -0.45 & +48 \\
\hline $\mathrm{FEV}_{1} / \mathrm{FVC}$ & 0.60 & 0.7 & NR & -3.15 & 0.69 & NR & -1.66 & +15 \\
\hline TLC (L) & 7.81 & 6.6 & 101 & NR & 7.60 & 99 & NR & -3 \\
\hline $\mathrm{RV}(\mathrm{L})$ & 3.21 & $3.0^{*}$ & 141 & NR & 1.72 & 76 & NR & -46 \\
\hline RV/TLC & 0.41 & $0.4^{*}$ & 124 & NR & 0.23 & 70 & NR & -44 \\
\hline $\mathrm{sG}_{\mathrm{aw}}\left(\left[(\mathrm{L} / \mathrm{s}) / \mathrm{cm} \mathrm{H}_{2} \mathrm{O}\right] / \mathrm{L}\right)$ & 0.05 & 0.1 & NR & NR & 0.15 & NR & NR & +200 \\
\hline $\begin{array}{l}\text { * Upper limit of the normal range. } \\
\mathrm{TLC}=\text { total lung capacity } \\
\mathrm{RV}=\text { residual volume } \\
\mathrm{sG}_{\text {aw }}=\text { specific airway conductance } \\
\mathrm{NR}=\text { not reported }\end{array}$ & & & & & & & & \\
\hline
\end{tabular}

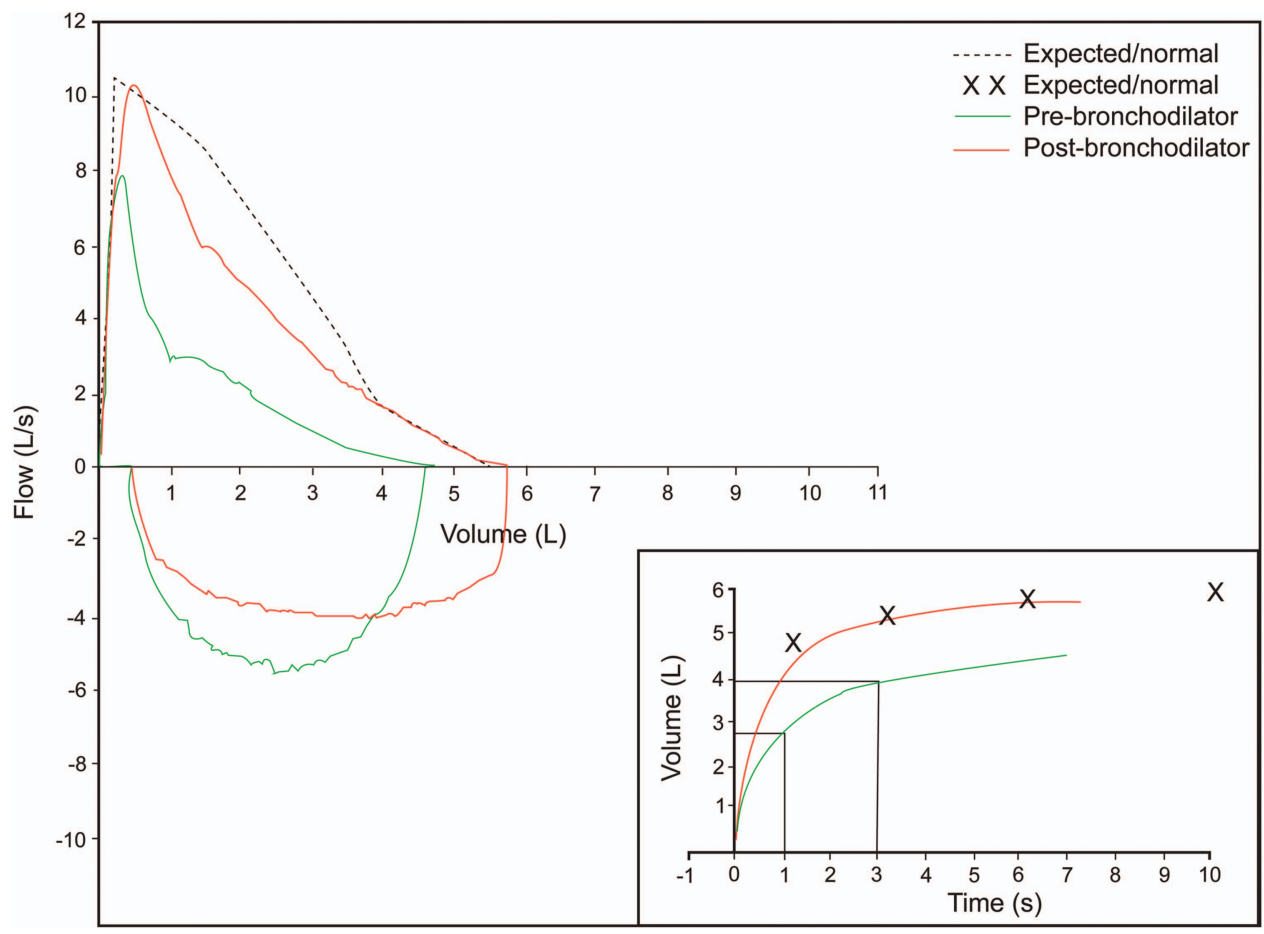

Fig. 2. Pre-bronchodilator and post-bronchodilator flow-volume loops and volume-time curves.

for the potential effects of deep inhalation during spirometry testing may lead to incorrect conclusions regarding the functionality of instruments and the quality of patient test performance. Moreover, the use of a dosimeter methacholine challenge test protocol that encourages inhalation to total lung capacity can result in false-negative challenge tests. ${ }^{5,6}$ The obvious danger of any false-negative diagnostic test is misdiagnosis and a misguided treatment plan.

In this case, an asthma patient (as determined by posttest probability) exhibited bronchoconstriction as a consequence of the deep inhalation necessary to perform forced spirometry. Historically, this phenomenon has been attributed to deep inhalation and not the expiratory phase of the maneuver. Moore et $\mathrm{al}^{14}$ showed no difference in response to methacholine between challenge tests that incorporated repeated exhalation to residual volume (without deep inhalation) and those that prohibited both deep inhalation and forced exhalation. In contrast, Suzuki et al ${ }^{15}$ showed a decline in $\mathrm{sG}_{\mathrm{aw}}$ following an expiratory maneuver from functional residual capacity; however, the decline associated with expiration was smaller than the decline associated with deep inhalation. 


\section{Bronchoconstriction During SpIROMEtry Testing}
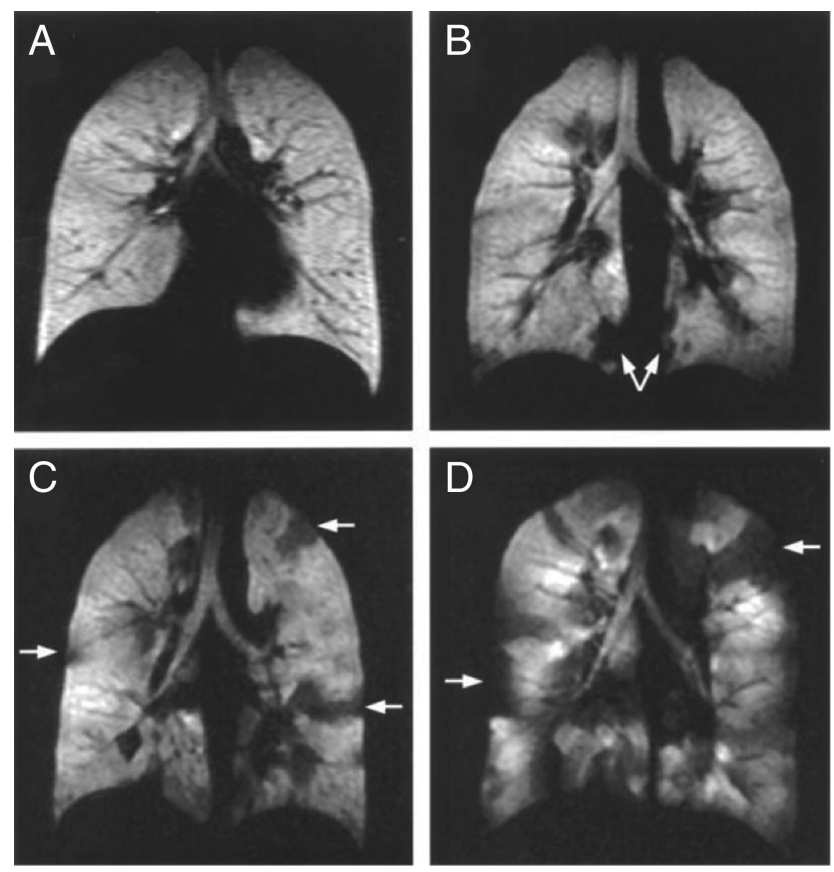

Fig. 3. Magnetic resonance ventilation images after inhalation of hyperpolarized helium. A: Normal volunteer with homogeneous distribution of ventilation. B: Mild asthma. C: Moderate asthma. D: Severe asthma. Arrows indicate ventilation defects. (From Reference 16, with permission.)

The exact mechanism of deep inhalation-induced bronchoconstriction is not completely understood and is presumably multifactorial. Our understanding of the very nature of air-flow obstruction in asthma has been greatly impacted by relatively recent advances in imaging techniques. These imaging techniques have shown that the classic model of relatively diffuse, homogeneous, and predictable patterns of bronchoconstriction are false. In fact, the asthmatic response is one of dynamic heterogeneous bronchoconstriction and bronchodilation, which are accompanied by patchy zones of parenchymal hypo-expansion and hyper-expansion, respectively. ${ }^{16-20}$ These zones of constricted airways and hypo-expanded parenchyma are referred to as ventilation defects (Fig. 3). ${ }^{16,20}$ Through radial traction as a function of airway parenchymal interdependence, the areas neighboring ventilation defects tend to experience bronchodilation and relative parenchymal hyper-expansion. Repeated deep inhalation, especially with limited volume, may be insufficient to expand the ventilation defects, leading to preferential ventilation of the already hyper-expanded zones..$^{20}$ This may cause unstable ventilation-defect boundary regions to collapse, resulting in larger and more numerous ventilation defects.

Burns and Gibson ${ }^{9}$ theorized that deep inhalation in the presence of obstruction might cause large swings in intrathoracic pressure, resulting in airway edema and worsening airway smooth muscle shortening. To test their the- ory, they measured $\mathrm{sG}_{\mathrm{aw}}$ in subjects with asthma and without asthma after deep inhalation with and without a resistive element in situ. Only the subjects with asthma demonstrated significant decreases in $\mathrm{sG}_{\mathrm{aw}}$ after deep inhalation with added resistance. Lim et al ${ }^{10}$ suggested that repeated deep inhalation has the potential to worsen airflow obstruction as a consequence of reduced deflation recoil. These data should prompt clinicians to exercise caution when asking acutely ill asthma patients to repeatedly perform deep inhalation for spirometry testing, peakflow measurements, and auscultation.

\section{Teaching Points}

- Deep inhalation have the potential to cause bronchoconstriction during spirometry testing.

- Careful observations are necessary to distinguish this phenomenon from instrument malfunction and suboptimal patient effort and technique.

- When bronchoconstriction is observed during spirometry testing, reporting the highest values as the best values may be misleading, especially when these values are linked to tests that are performed after the bronchoconstriction has occurred. The best approach is to show all of the data to the interpreting physician.

- Clinicians should exercise caution when asking acutely ill asthma patients to repeatedly perform deep inhalation for spirometry testing, peak-flow measurements, and auscultation.

\section{REFERENCES}

1. Kaminsky DA. What does airway resistance tell us about lung function? Respir Care 2012;57(1):85-96; discussion 96-99.

2. Fish JE, Ankin MG, Kelly JF, Peterman VI. Regulation of bronchomotor tone by lung inflation in asthmatic and nonasthmatic subjects. J Appl Physiol Respir Environ Exerc Physiol 1981;50(5):10791086.

3. Fredberg JJ, Inouye D, Miller B, Nathan M, Jafari S, Raboudi SH, et al. Airway smooth muscle, tidal stretches, and dynamically determined contractile states. Am J Respir Crit Care Med 1997;156(6): 1752-1759.

4. Gump A, Haughney L, Fredberg J. Relaxation of activated airway smooth muscle: relative potency of isoproterenol vs. tidal stretch. J Appl Physiol 2001;90(6):2306-2310.

5. Cockcroft DW, Davis BE. The bronchoprotective effect of inhaling methacholine by using total lung capacity inspirations has a marked influence on the interpretation of the test result. J Allergy Clin Immunol 2006;117(6):1244-1248.

6. Allen ND, Davis BE, Hurst TS, Cockcroft DW. Difference between dosimeter and tidal breathing methacholine challenge: contributions of dose and deep inspiration bronchoprotection. Chest 2005;128(6): 4018-4023.

7. Skloot G, Permutt S, Togias A. Airway hyperresponsiveness in asthma: a problem of limited smooth muscle relaxation with inspiration. J Clin Invest 1995;96(5):2393-2403. 


\section{Bronchoconstriction During SpIROMEtry Testing}

8. Burns GP, Gibson GJ. Airway hyperresponsiveness in asthma. Not just a problem of smooth muscle relaxation with inspiration. Am J Respir Crit Care Med 1998;158(1):203-206.

9. Burns GP, Gibson GJ. A novel hypothesis to explain the bronchoconstrictor effect of deep inspiration in asthma. Thorax 2002;57(2): 116-119.

10. Lim TK, Ang SM, Rossing TH, Ingenito EP, Ingram RH. The effects of deep inhalation on maximal expiratory flow during intensive treatment of spontaneous asthmatic episodes. Am Rev Respir Dis 1989; 140(2):340-343.

11. Quanjer PH, Stanojevic S, Cole TJ, Baur X, Hall GL, Culver BH, et al. Multi-ethnic reference values for spirometry for the 3-95-yr age range: the global lung function 2012 equations. Eur Respir J 2012; 40(6): 1324-1343.

12. Miller MR, Hankinson J, Brusasco V, Burgos F, Casaburi R, Coates A, et al. Standardisation of spirometry. Eur Respir J 2005;26(2):319338.

13. Bates JH. Lung mechanics, an inverse modeling approach. New York: Cambridge University Press; 2009:9-14.

14. Moore BJ, Verburgt LM, King GG, Paré PD. The effect of deep inspiration on methacholine dose-response curves in normal subjects. Am J Respir Crit Care Med 1997;156(4 Pt 1):1278-1281.
15. Suzuki S, Miyashita A, Matsumoto Y, Okubo T. Bronchoconstriction induced by spirometric maneuvers in patients with bronchial asthma. Ann Allergy 1990;65(4):315-320.

16. Samee S, Altes T, Powers P, de Lange EE, Knight-Scott J, Rakes G, et al. Imaging the lungs in asthmatic patients by using hyperpolarized helium-3 magnetic resonance: assessment of response to methacholine and exercise challenge. J Allergy Clin Immunol 2003;111(6): 1205-1211.

17. Brown RH, Scichilone N, Mudge B, Diemer FB, Permutt S, Togias A. High-resolution computed tomographic evaluation of airway distensibility and the effects of lung inflation on airway caliber in healthy subjects and individuals with asthma. Am J Respir Crit Care Med 2001;163(4):994-1001.

18. Venegas JG, Winkler T, Musch G, Vidal Melo MF, Layfield D, Tgavalekos N, et al. Self-organized patchiness in asthma as a prelude to catastrophic shifts. Nature 2005;434(7034):777-782.

19. Tgavalekos NT, Musch G, Harris RS, Vidal Melo MF, Winkler T, Schroeder T, et al. Relationship between airway narrowing, patchy ventilation and lung mechanics in asthmatics. Eur Respir J 2007; 29(6):1174-1181.

20. Golnabi AH, Harris RS, Venegas JG, Winkler T. Deep inspiration and the emergence of ventilation defects during bronchoconstriction: a computational study. PLoS ONE 2014;9(11):e112443. 